\title{
Compact MILP formulations for the $p$-center problem
}

\author{
Zacharie Ales ${ }^{1}$, Sourour Elloumi ${ }^{1}$ \\ ENSTA-ParisTech / UMA, 91762 Palaiseau, France \\ Laboratoire CEDRIC, Paris, France \\ \{zacharie.ales, sourour.elloumi\}@ensta-paristech.fr
}

Keywords: $p$-center, discrete location, equivalent formulations, integer programming.

\begin{abstract}
The $p$-center problem consists in selecting $p$ centers among $M$ to cover $N$ clients, such that the maximal distance between a client and its closest selected center is minimized. For this problem we propose two new and compact integer formulations.

Our first formulation is an improvement of a previous formulation. It significantly decreases the number of constraints while preserving the optimal value of the linear relaxation. Our second formulation contains less variables and constraints but it has a weaker linear relaxation bound. We besides introduce an algorithm which enables us to compute strong bounds and significantly reduce the size of our formulations.

Finally, the efficiency of the algorithm and the proposed formulations are compared in terms of quality of the linear relaxation and computation time over instances from OR-Library.
\end{abstract}

\section{Introduction}

We consider $N$ clients $\left\{C_{1}, \ldots, C_{N}\right\}$ and $M$ potential facility sites $\left\{F_{1}, \ldots, F_{M}\right\}$. Let $d_{i j}$ be the distance between $C_{i}$ and $F_{j}$. The objective of the $p$-center problem is to open up to $p$ facilities such that the maximal distance (called radius) between a client and its closest selected site is minimized. This problem is very popular in combinatorial optimization and has many applications. We refer the reader to the recent survey [2]. Very recent publications include $[7,6]$ which provide heuristic solutions and $[3]$ on an exact solution method.

In this paper, we will focus on mixed-integer linear programming formulations of the $p$-center problem.

Let $\mathcal{M}$ and $\mathcal{N}$ respectively be the sets $\{1, \ldots, M\}$ and $\{1, \ldots, N\}$. The most classical formulation, denoted by $\left(P_{1}\right)$, for the $p$-center problem (see for example [4]) considers the following variables:

$-y_{j}$ is a binary variable equal to 1 if and only if $F_{j}$ is open;

- $x_{i j}$ is a binary variable equal to 1 if and only if $C_{i}$ is assigned to $F_{j}$;

$-R$ is the radius. 


$$
\left(P_{1}\right)\left\{\begin{array}{rr}
\min R & \\
\text { s.t. } \sum_{j=1}^{M} y_{j} \leq p & i \in \mathcal{N} \\
\sum_{j=1}^{M} x_{i j}=1 & i \in \mathcal{N}, j \in \mathcal{M} \\
x_{i j} \leq y_{j} & i \in \mathcal{N} \\
\sum_{j=1}^{M} d_{i j} x_{i j} \leq R & i \in \mathcal{N}, j \in \mathcal{M} \\
x_{i j}, y_{j} \in\{0,1\} & \\
r \in \mathbb{R} &
\end{array}\right.
$$

Constraint (1b) ensures that no more than $p$ facilities are opened. Each client is assigned to exactly one facility through Constraints (1c). Constraints (1d) link variables $x_{i j}$ and $y_{j}$ while (1e) ensure the coherence of the objective.

A more recent formulation, denoted by $\left(P_{2}\right)$, was proposed in [5]. Let $D^{0}<D^{1}<\ldots<D^{K}$ be the different $d_{i j}$ values $\forall i \in \mathcal{N} \forall j \in \mathcal{M}$. Note that, if many distances $d_{i j}$ have the same value, $K$ may be significantly lower than $M \times N$. Let $\mathcal{K}$ be the set $\{1, \ldots, K\}$. Formulation $\left(P_{2}\right)$ is based on the variables $y_{j}$, previously introduced, and one binary variable $z^{k}$, for each $k \in \mathcal{K}$, equals to 1 if and only if the optimal radius is greater than or equal to $D^{k}$ :

$$
\left(P_{2}\right)\left\{\begin{array}{cc}
\min D^{0}+\sum_{k=1}^{K}\left(D^{k}-D^{k-1}\right) z^{k} & \\
\text { s.t. } 1 \leq \sum_{j=1}^{M} y_{j} \leq p & \\
z^{k}+\sum_{j: d_{i j}<D^{k}} y_{j} \geq 1 & i \in \mathcal{N}, k \in \mathcal{K} \\
y_{j}, z^{k} \in\{0,1\} & j \in \mathcal{M}, k \in \mathcal{K}
\end{array}\right.
$$

Constraints (2c) ensure that if no facility located at less than $D^{k}$ of client $C_{i}$ is selected, then the radius must be greater than or equal to $D^{k}$.

This formulation has been proved to be tighter than $\left(P_{1}\right)$ [5]. However, its size strongly depends on the value $K$ (i.e., the number of distinct distances $d_{i j}$ ).

It also has recently been adapted to the $p$-dispersion problem which consists in selecting $p$ facilities among $N$ such that the minimal distance between two selected facilities is maximized [8].

A last formulation, that can be deduced from $\left(P_{2}\right)$ by a change of variables, has been recently introduced [3] and named $\left(P_{4}\right)$. It contains, for all $k \in \mathcal{K}$, a binary variable $u_{k}$ equal to 1 if and only if the optimal radius is $D^{k}$ (i.e., $u_{k}=z^{k}-z^{k+1}$ and $z^{k}=\sum_{q=k}^{K} u_{q}$ ): 


$$
\left(P_{4}\right)\left\{\begin{array}{l}
\min \sum_{k=1}^{K} D^{k} u_{k} \\
\text { s.t. }(2 \mathrm{~b}) \\
\sum_{j: d_{i j} \leq D^{k}} y_{j} \geq \sum_{q=1}^{k} u_{q} \\
\sum_{k=1}^{K} u_{k}=1 \\
y_{j}, u_{k} \in\{0,1\} \quad i \in \mathcal{N}, k \in \mathcal{K}
\end{array}\right.
$$

They also proposed a weaker version of this formulation, called $\left(P_{3}\right)$, obtained by replacing the left-hand side of constraints $(3 \mathrm{~b})$ by $u_{k}$. They proved that $\left(P_{4}\right)$ leads to the same linear relaxation bound and has the same size as $\left(P_{2}\right)$.

The rest of the paper is organized as follows. Section 2 presents our two new formulations. In Section 3 we introduce an algorithm. Finally, Section 4 describes numerical results on instances from the OR-Library.

\section{Our new formulations}

\subsection{Formulation $\left(C P_{1}\right)$}

In $\left(P_{2}\right)$, for all $k \in \mathcal{K}$, variable $z^{k}$ is equal to 1 if and only if the optimal radius is greater than or equal to $D^{k}$. As a consequence, the following constraints are valid

$$
z^{k} \geq z^{k+1} \quad k \in\{1, \ldots, K-1\} .
$$

We first show that these inequalities are redundant for $\left(P_{2}\right)$. Let $\left(P_{2}^{\prime}\right)$ be the formulation obtained when contraints $(4)$ are added to $\left(P_{2}\right)$ and let $v(\bar{F})$ be the optimal value of the linear relaxation of a given formulation $F$. We now prove that adding constraints (4) does not improve the quality of the linear relaxation.

Proposition 1. $v\left(\overline{P_{2}^{\prime}}\right)=v\left(\overline{P_{2}}\right)$

Proof. We show that an optimal solution $(\tilde{y}, \tilde{z})$ of the relaxation of $\left(P_{2}\right)$ satisfies $(4)$. For each distance $D^{k}$ there exists a client $i(k)$ such that

$$
\tilde{z}^{k}+\sum_{j: d_{i(k) j}<D^{k}} \tilde{y}_{j}=1
$$

otherwise $\tilde{z}^{k}$ can be decreased and $(\tilde{y}, \tilde{z})$ is not optimal.

We now assume that $\tilde{z}^{k-1}<\tilde{z}^{k}$ for some index $k \in\{2, \ldots, K\}$. It follows that

$$
\tilde{z}^{k-1}+\sum_{j: d_{i(k) j}<D^{k-1}} \tilde{y}_{j}<\tilde{z}^{k}+\sum_{j: d_{i(k) j}<D^{k}} \tilde{y}_{j}=1
$$


The last equality follows from (5). Therefore, constraints (2c) for $i(k)$ and $k-1$ is violated.

We now prove that a large part of constraints $(2 \mathrm{c})$ are redundant in $\left(P_{2}^{\prime}\right)$. Let $N_{i}^{k}$ be the set of facilities located at less than $D^{k}$ from client $C_{i}$. We can observe that $N_{i}^{k}$ is included in $N_{i}^{k+1}$, for all $k \in \mathcal{K}$. Moreover, $N_{i}^{k}$ is equal to $N_{i}^{k+1}$ if and only if there is no facility at distance $D^{k}$ from client $C_{i}$. Let $S_{i}$ be the set of indices $k \in\{1, \ldots, K-1\}$ such that $N_{i}^{k}$ is different from $N_{i}^{k+1}$. Observe that $\left|S_{i}\right| \leq \min (M, K)$.

We define Formulation $\left(C P_{1}\right)$ as Formulation $\left(P_{2}^{\prime}\right)$ where only the constraints (2c) such that $k \in S_{i}$ or $k=K$ are kept.

$$
\left(C P_{1}\right)\left\{\begin{array}{cr}
\min D^{0}+\sum_{k=1}^{K}\left(D^{k}-D^{k-1}\right) z^{k} & \\
\text { s.t. }(2 \mathrm{~b}),(4) & \\
z^{k}+\sum_{j: d_{i j}<D^{k}} y_{j} \geq 1 & i \in \mathcal{N}, \quad k \in S_{i} \cup\{K\} \\
y_{j}, z^{k} \in\{0,1\} & j \in \mathcal{M}, k \in \mathcal{K}
\end{array}\right.
$$

The number of constraints is dominated by the number of constraints $(6 \mathrm{~b})$. This number is bounded by both $N M$ and $N K$.

The following proposition proves that $\left(C P_{1}\right)$ is a valid formulation.

Proposition 2. $\left(C P_{1}\right)$ is a valid formulation of the p-center problem.

Proof. We show that the constraints removed from $\left(P_{2}^{\prime}\right)$ are dominated. If $N_{i}^{k}=N_{i}^{k+1}$, then $\sum_{j: d_{i j}<D^{k}} y_{j}=\sum_{j: d_{i j}<D^{k+1}} y_{j}$. Since $z^{k} \geq z^{k+1}$, we have:

$$
z^{k}+\sum_{j: d_{i j}<D^{k}} y_{j} \geq z^{k+1}+\sum_{j: d_{i j}<D^{k+1}} y_{j} \geq 1 .
$$

As a consequence, the constraint (2c) associated with $i$ and $k$ is dominated by the one associated with $i$ and $k+1$.

We now prove that Formulations $\left(P_{2}\right)$ and $\left(C P_{1}\right)$ lead to the same bound by linear relaxation.

Proposition 3. $v\left(\overline{C P_{1}}\right)=v\left(\overline{P_{2}}\right)$.

Proof. The arguments used in the proof of Proposition 2 can be used again to show that the constraints removed from $\left(P_{2}^{\prime}\right)$ do not impact the value of the linear relaxation.

To sum up, $\left(C P_{1}\right)$ is a valid formulation that has the same LP bound as $\left(P_{2}\right)$. However, as detailed in Table 1 , Formulation $\left(C P_{1}\right)$ is much smaller since it reduces the number of constraints by a factor of up to $N$. 


\subsection{Formulation $\left(\mathrm{CP}_{2}\right)$}

We now introduce a second formulation, denoted by $\left(C P_{2}\right)$, which contains less variables and constraints than $\left(C P_{1}\right)$.

We replace the $K$ binary variable $z^{k}$ with a unique general integer variable $r$ which represents the index of a radius:

$$
\left(C P_{2}\right)\left\{\begin{array}{ll}
\min r & \\
\text { s.t. }(2 \mathrm{~b}) & \\
r+k \sum_{j: d_{i j}<D^{k}} y_{j} \geq k \quad i \in \mathcal{N}, k \in S_{i} \cup\{K\} \\
y_{j} \in\{0,1\} \\
r \in\{0, \ldots, K\}
\end{array} \quad j \in \mathcal{M}\right.
$$

Constraints (7a) play a similar role to Constraints (6b).

Formulation $\left(\mathrm{CP}_{2}\right)$ does not directly provide the value of the optimal radius $R$ but its index $r$ such that $D^{r}=R$. We now prove that Formulation $\left(C P_{2}\right)$ is valid.

Proposition 4. $\left(\mathrm{CP}_{2}\right)$ is a valid formulation of the p-center problem.

Proof. Let $(\tilde{y}, \tilde{z})$ be an integer solution of $\left(C P_{1}\right)$. We first show that there exists an integer solution $(\bar{y}, \bar{r})$ of $\left(C P_{2}\right)$ which provides the same radius by setting $\bar{y}=\tilde{y}$ and $\bar{r}=\sum_{k=1}^{K} \tilde{z}^{k}$. We need to prove that constraints (7a) are satisfied. We know that

$$
\tilde{z}^{k}+\sum_{j: d_{i j}<D^{k}} \tilde{y}_{j} \geq 1
$$

is satisfied for any client $C_{i}$ and any distance $D^{k}$.

If $\tilde{z}^{k}$ is equal to 0 , the corresponding Constraint (7a) is satisfied, as $\sum_{j: d_{i j}<D^{k}} \tilde{y}_{j} \geq 1$. Otherwise, the same result is obtained since the $\tilde{z}^{k}$ variables are ordered in decreasing order which leads to $\bar{r} \geq k$. These two solutions provide the same radius as $D^{0}+\sum_{k=1}^{K}\left(D^{k}-\bar{D}^{k-1}\right) \tilde{z}^{k}=$ $D^{\sum_{k=1}^{K} \tilde{z}^{k}}$.

We now prove that for any solution $(\tilde{y}, \tilde{r})$ of $\left(C P_{2}\right)$ there exists an equivalent solution $(\bar{y}, \bar{z})$ of $\left(C P_{1}\right)$. We set $\bar{y}=\tilde{y}$ and $\bar{z}^{k}=1$ if and only if $\tilde{r} \geq k$. Constraint

$$
\tilde{r}+k \sum_{j: d_{i j}<D^{k}} \tilde{y}_{j} \geq k
$$

is satisfied for any $k \in \mathcal{K}$. If $\tilde{r}$ is lower than $k$, then at least one variable $\tilde{y}_{j}$ from equation (8) is equal to 1 and the corresponding constraint (6b) is satisfied. Otherwise, $\bar{z}^{k}$ is equal to 1 and the same conclusion is reached.

We now prove that the linear relaxation of $\left(C P_{1}\right)$ is stronger than the one of $\left(C P_{2}\right)$. 
Assumption 1 We shall suppose $D^{0}=0$ and $\forall k \in \mathcal{K}, D^{k}-D^{k-1}=1$.

This assumption is not restrictive, one can transform any instance by replacing any distance $D^{k}$ by its rank $k$. The transformed problem is equivalent as if the optimal radius is $D^{k^{*}}$, then the optimal solution of the transformed problem is $k^{*}$.

Under this assumption, problems $\left(C P_{1}\right)$ and $\left(C P_{2}\right)$ have the same optimal values, both of them compute the rank of the optimal radius.

Proposition 5. Let $\overline{C P_{1}}$ and $\overline{C P_{2}}$ respectively be the $L P$ relaxation of $\left(C P_{1}\right)$ and $\left(C P_{2}\right), v\left(\overline{C P_{1}}\right) \geq v\left(\overline{C P_{2}}\right)$ under Assumption 1.

Proof. Let $(\tilde{y}, \tilde{z})$ be a solution of $\overline{C P_{1}}$. We build a solution $(\bar{y}, \bar{r})$ of $\overline{C P_{2}}$ with the same value. We take $\bar{y}=\tilde{y}$ and $\bar{r}=\sum_{k=1}^{K} \tilde{z}^{k}$.

We need to prove that constraints (7a) are satisfied.

Since the $z^{k}$ variables are ordered in decreasing order by Constraints 4 , it follows that $\bar{r} \geq k \tilde{z}^{k} \forall k \in \mathcal{K}$. This and Constraints (2c) imply that Constraints (7a) are satisfied.

Table 1 summarizes the size of the previously mentioned formulations.

\begin{tabular}{ccc}
\hline Formulation & \# of variables & \# of constraints \\
\hline$\left(P_{1}\right)$ & $\mathcal{O}(N M)$ & $\mathcal{O}(N M)$ \\
$\left(P_{2}\right),\left(P_{3}\right),\left(P_{4}\right)$ & $\mathcal{O}(M+K)$ & $\mathcal{O}(N K)$ \\
$\left(C P_{1}\right)$ & $\mathcal{O}(M+K)$ & $\mathcal{O}(\min (N M, N K))$ \\
$\left(C P_{2}\right)$ & $\mathcal{O}(M)$ & $\mathcal{O}(\min (N M, N K))$ \\
\hline
\end{tabular}

Table 1. Size of the four formulations $(K \leq N M)$.

\section{A two-step resolution algorithm}

We present, in this section, a two-step algorithm to solve more efficiently the $p$-center problem.

Let $l b$ be a lower bound of the optimal radius. We suppose that $l b$ is one of the distances $D^{k}$ since, otherwise, $l b$ can be set to the next distance. All the distances $d_{i j}$ lower than $l b$ can be replaced by $l b$.

Similarly, all the distances $d_{i j}$ greater than an upper bound $u b$ can be replaced by $u b+1$ in order to discard solutions of value greater than $u b$. The size of Formulations $\left(P_{2}\right)$ and $\left(C P_{1}\right)$ strongly depends on $K$. This value can be reduced by identifying lower and upper bounds. Such bounds can easily be obtained, as mentioned in [5]. 
Our resolution algorithm, depicted in Figure 1, can be applied to any formulation $F$ of the $p$-center problem including $\left(P_{1}\right),\left(P_{2}\right),\left(P_{3}\right),\left(P_{4}\right)$, $\left(C P_{1}\right)$ and $\left(C P_{2}\right)$. It is mainly based on the idea that whenever the optimal value $\bar{v}$ of the linear relaxation of $F$ is not equal to an existing distance, then there exists $k \in K$ such that $D^{k-1}<\bar{v}<D^{k}$. In that case, $D^{k}$ constitutes a stronger lower bound than $\bar{v}$ and the linear relaxation can be solved again. This process is repeated until an existing distance is obtained as the optimal value of the linear relaxation. This constitutes Step 1 of the algorithm.

The bound obtained when applying this algorithm over $\left(P_{2}\right)$ or $\left(C P_{1}\right)$ corresponds to the one called $L B^{*}$, computed by a binary search algorithm in [5].

Step 1 can be further improved by introducing the notion of dominated clients and dominated facilities within some reduction rules. A facility $F_{a}$ is dominated if there exists another facility $F_{b}$ such that $d_{i a} \geq d_{i b}$ for all clients $i$. Such a facility can be removed as it will always be at least as interesting to assign a client to $F_{b}$ than to $F_{a}$. Similarly, a client $C_{a}$ is said to be dominated if there exists another client $C_{b}$ such that $d_{a j} \leq d_{b j}$ for all facilities $j$. Dominated clients can also be ignored.

Instructions 3 and 4 are repeated since new dominated clients and facilities may be found when a bound is improved, and vice versa.

Step 2 of Algorithm 1 consists in solving Formulation $F$ to optimality with the improved bounds $l b$ and $u b$ computed in Step 1.

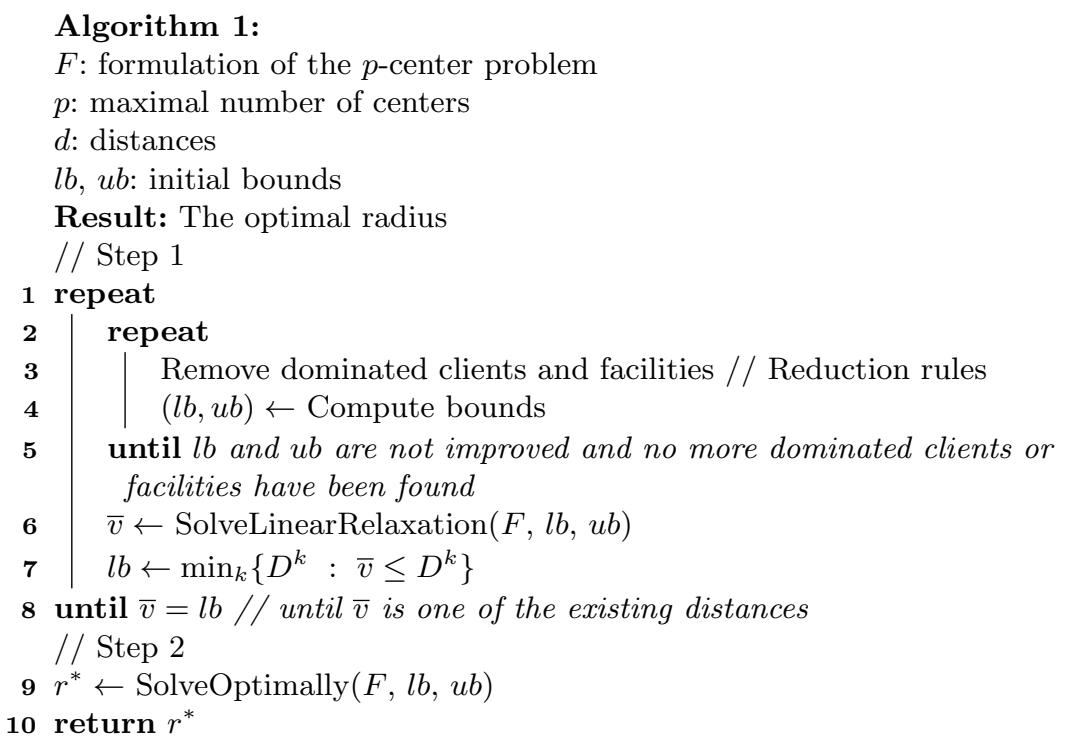

Fig. 1. Algorithm used to solve the $p$-center problem through $\mathrm{F}$, a $p$-center formulation. 


\section{Numerical results}

We implement Formulations $\left(P_{1}\right),\left(P_{2}\right),\left(C P_{1}\right)$ and $\left(C P_{2}\right)$ as well as Algorithm 1 on an Intel XEON E3-1280 with 3,5 GHz and 32Go of RAM with the Java API of CPLEX 12.7. Following several authors, we consider instances from the OR-Library [1].

\subsection{Comparing sizes and computation times on 5 instances}

Table 2 presents a comparison of the sizes of the four formulations on the five first instances of the OR-Library with $N=M=100$. We use the initial lower bound $L B_{0}=\max _{i \in \mathcal{N}} \min _{j \in \mathcal{M}} d_{i j}$ and initial upper bound $U B_{0}=\min _{j \in \mathcal{M}} \max _{i \in \mathcal{N}} d_{i j}$ introduced in [5].

As expected, the number of variables in $\left(C P_{1}\right)$ and $\left(P_{2}\right)$ are equal and are significantly lower than in $\left(P_{1}\right)$. Formulation $\left(P_{2}\right)$ has more constraints than Formulation $\left(P_{1}\right)$. Formulation $\left(C P_{1}\right)$ has by far less constraints than $\left(P_{2}\right)$. All this explains why $\left(C P_{1}\right)$ has the best performances in every aspect.

Formulation $\left(\mathrm{CP}_{2}\right)$ is the most compact but this does not fully compensate the poor quality of its LP bound.

\begin{tabular}{l|lrrrr}
\hline & & $\left(\mathbf{P}_{\mathbf{1}}\right)$ & $\left(\mathbf{P}_{\mathbf{2}}\right)$ & $\left(\mathbf{C P}_{\mathbf{1}}\right)$ & $\left(\mathbf{C P}_{\mathbf{2}}\right)$ \\
\hline Instance 1 & number of variables & 10101 & 286 & 286 & 101 \\
& number of constraints & 12209 & 18602 & 6089 & 5903 \\
$\left(L B_{0}=0\right)$ & LP bound & 97,57 & 106,54 & 106,54 & 83,62 \\
$\left(U B_{0}=186\right)$ & resolution time $(\mathrm{s})$ & 9,14 & 251,28 & $\mathbf{3 , 1 6}$ & 14,94 \\
\hline Instance 2 & number of variables & 10101 & 277 & 277 & 101 \\
& number of constraints & 12473 & 17702 & 6094 & 5917 \\
$\left(L B_{0}=0\right)$ & LP bound & 76,72 & 85,68 & 85,68 & 70,19 \\
$\left(U B_{0}=178\right)$ & resolution time $(\mathrm{s})$ & 15,69 & 47,31 & $\mathbf{2 , 9 9}$ & 19,80 \\
\hline Instance 3 & number of variables & 10101 & 305 & 305 & 101 \\
& number of constraints & 11293 & 20502 & 6852 & 6647 \\
$\left(L B_{0}=0\right)$ & LP bound & 73,24 & 83,28 & 83,28 & 68,92 \\
$\left(U B_{0}=205\right)$ & resolution time $(\mathrm{s})$ & 11,68 & 21,02 & $\mathbf{2 , 8 5}$ & 10,99 \\
\hline Instance 4 & number of variables & 10101 & 299 & 299 & 101 \\
& number of constraints & 12009 & 19902 & 6403 & 6204 \\
$\left(L B_{0}=0\right)$ & LP bound & 54,55 & 64,16 & 64,16 & 52,42 \\
$\left(U B_{0}=204\right)$ & resolution time $(\mathrm{s})$ & 3,19 & 43,02 & $\mathbf{1 , 6 4}$ & 12,90 \\
\hline Instance 5 & number of variables & 10101 & 270 & 270 & 101 \\
& number of constraints & 11777 & 17002 & 6263 & 6093 \\
$\left(L B_{0}=0\right)$ & LP bound & 30,37 & 37,82 & 37,82 & 29,29 \\
$\left(U B_{0}=169\right)$ & resolution time $(\mathrm{s})$ & 1,93 & 25,10 & $\mathbf{1 , 6 6}$ & 11,65 \\
\hline
\end{tabular}

Table 2. Size and resolution times (1 thread) of the formulations for the five first OR-Library instances with $l b=L B_{0}$ and $u b=U B_{0}$. 


\subsection{Relaxation and computation times on the 40 OR-Library instances}

In Table 3, we perform a larger comparison with stronger bounds $l b$ and $u b$ equal to the bounds $L B_{1}$ and $U B_{1}$ introduced in [5]. The resolution is then performed by CPLEX with its default parameters but with a maximal CPU time of 1 hour.

The first column is the instance number. The three following columns provide $N, p$ and the optimal value of the instances $(N=M$ in these instances). Columns 5 and 6 contain the initial bounds $L B$ and $U B$. For each formulation, column " $b$ " corresponds the optimal value of the linear relaxation and column " $\mathrm{t}$ " to the resolution time in seconds.

We can first observe that Formulations $\left(C P_{1}\right)$ and $\left(P_{2}\right)$ solve all the 40 instances within 1 hour while ten instances are not solved with $\left(P_{1}\right)$ and one instance is not solved with $\left(C P_{2}\right)$. We can even observe that $\left(C P_{1}\right)$ solves the whole set of instances in less than 50 minutes and $\left(P_{2}\right)$ in less than 85 minutes.

Formulation $\left(P_{2}\right)$ outperforms $\left(C P_{1}\right)$ mainly on instances 36 and 39 . This is possibly due to some difficulty of the solver to find good feasible solutions.

\subsection{Results of Algorithm 1}

Table 4 presents the results of Algorithm 1 with formulations $\left(C P_{1}\right)$ and $\left(C P_{2}\right)$. Columns "t1" and "t2" respectively correspond to the time of the first phase and the total time.

Formulation $\left(\mathrm{CP}_{2}\right)$ is now able to solve all the instances within 1 hour. We observe that the total time to solve the 40 instances is reduced by approximately 6 times for $\left(C P_{1}\right)$ and 14 times for $\left(C P_{2}\right)$ if compared to Table 3.

\section{Conclusion}

We introduced two new compact formulations of the $p$-center problem. We theoretically compared the quality of their LP bounds and their sizes to existing formulations. Numerical experiments confirmed these results and highlighted the fact that our new formulation $\left(C P_{1}\right)$ outperforms the previously known formulations $\left(P_{1}\right)$ and $\left(P_{2}\right)$ at all levels. Our more compact formulation $\left(\mathrm{CP}_{2}\right)$ suffers from the poor quality of its linear relaxation. Another aspect of our work was to embed the formulations within a two-step algorithm in order to obtain better computation times. Our future work will focus on improving our compact formulation through polyhedral studies.

\section{References}

1. John E. Beasley. Or-library: distributing test problems by electronic mail. Journal of the operational research society, pages 1069-1072, 1990. 


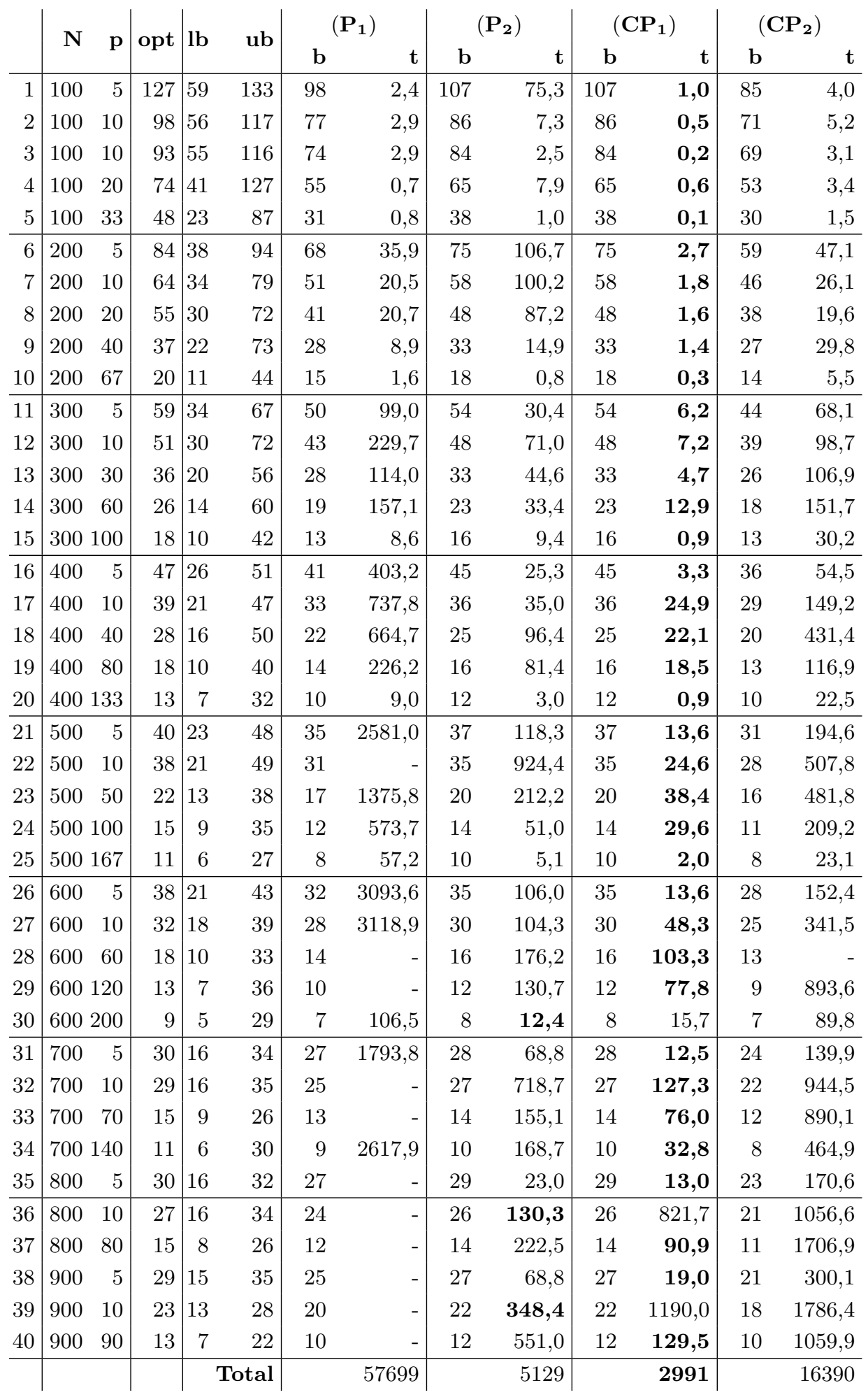

Table 3. Comparison of the different formulations with $l b=L B_{1}$ and $u b=U B_{1}$. For each instance, the smallest time appears in bold. Symbol "-" means that the instance was not solved within 1 hour. 


\begin{tabular}{|c|c|c|c|c|c|c|}
\hline & \multirow{2}{*}{$\mathbf{p}$} & \multirow{2}{*}{ opt } & \multicolumn{2}{|c|}{$\left(\mathbf{C P}_{\mathbf{1}}\right)$} & \multicolumn{2}{|c|}{$\left(\mathbf{C P}_{2}\right)$} \\
\hline & & & t1 & t2 & t1 & $\mathrm{t} 2$ \\
\hline 1100 & 5 & 127 & 0,2 & 0,3 & 0,3 & 0,7 \\
\hline 2100 & 10 & 98 & 0,2 & 0,2 & 0,3 & 0,4 \\
\hline 3100 & 10 & 93 & 0,2 & 0,3 & 0,3 & 0,4 \\
\hline 4100 & 20 & 74 & 0,3 & 0,4 & 0,4 & 0,5 \\
\hline 5100 & 33 & 48 & 0,1 & 0,2 & 0,3 & 0,4 \\
\hline 6200 & 5 & 84 & 1,9 & 2,7 & 5,2 & 6,3 \\
\hline 7200 & 10 & 64 & 1,1 & 1,4 & 3,0 & 3,4 \\
\hline 8200 & 20 & 55 & 0,8 & 1,0 & 2,8 & 3,0 \\
\hline 9200 & 40 & 37 & 2,0 & 2,7 & 4,5 & 5,4 \\
\hline 10200 & 67 & 20 & 0,4 & 0,6 & 0,9 & 1,1 \\
\hline 11300 & 5 & 59 & 0,8 & 0,9 & 2,2 & 2,2 \\
\hline 12300 & 10 & 51 & 3,4 & 4,6 & 10,2 & 12,5 \\
\hline 13300 & 30 & 36 & 3,6 & 4,6 & 8,8 & 9,8 \\
\hline 14300 & 60 & 26 & 3,5 & 4,5 & 14,8 & 17,5 \\
\hline 15300 & 100 & 18 & 1,5 & 2,1 & 3,3 & 3,7 \\
\hline 16400 & 5 & 47 & 1,4 & 1,4 & 6,4 & 6,4 \\
\hline 17400 & 10 & 39 & 3,3 & 4,3 & 9,5 & 10,6 \\
\hline 18400 & 40 & 28 & 5,8 & 8,3 & 29,1 & 33,3 \\
\hline 19400 & 80 & 18 & 4,1 & 6,2 & 9,8 & 12,1 \\
\hline 20400 & 133 & 13 & 2,5 & 3,0 & 4,0 & 5,0 \\
\hline 21500 & 5 & 40 & 3,1 & 4,0 & 9,7 & 10,3 \\
\hline 22500 & 10 & 38 & 16,6 & 26,5 & 38,6 & 48,3 \\
\hline 23500 & 50 & 22 & 7,0 & 9,9 & 31,5 & 37,1 \\
\hline 24500 & 100 & 15 & 7,6 & 11,4 & 18,5 & 23,7 \\
\hline 25500 & 167 & 11 & 3,7 & 4,6 & 7,5 & 9,0 \\
\hline 26600 & 5 & 38 & 4,6 & 5,3 & 19,3 & 20,7 \\
\hline 27600 & 10 & 32 & 9,5 & 12,5 & 23,0 & 26,2 \\
\hline 28600 & 60 & 18 & 14,4 & 17,5 & 42,0 & 48,7 \\
\hline 29600 & 120 & 13 & 23,4 & 32,7 & 91,0 & 111,4 \\
\hline 30600 & 200 & 9 & 10,5 & 15,1 & 17,4 & 21,9 \\
\hline 31700 & 5 & 30 & 8,2 & 9,3 & 15,8 & 17,5 \\
\hline 32700 & 10 & 29 & 18,8 & 71,8 & 33,8 & 109,8 \\
\hline 33700 & 70 & 15 & 10,2 & 14,3 & 25,4 & 34,4 \\
\hline 34700 & 140 & 11 & 34,2 & 46,4 & 90,1 & 107,6 \\
\hline 35800 & 5 & 30 & 2,2 & 2,2 & 11,8 & 12,0 \\
\hline 36800 & 10 & 27 & 20,0 & 30,3 & 40,5 & 53,1 \\
\hline 37800 & 80 & 15 & 21,8 & 27,8 & 50,2 & 60,9 \\
\hline 38900 & 5 & 29 & 12,2 & 12,7 & 29,7 & 30,3 \\
\hline 39900 & 10 & 23 & 36,6 & 49,7 & 45,5 & 153,4 \\
\hline 40900 & 90 & 13 & 21,8 & 31,2 & 50,3 & 70,7 \\
\hline Total & & & & 484 & & 1142 \\
\hline
\end{tabular}

Table 4. Results obtained with Algorithm 1 of Figure 1 with $l b=L B_{1}$ and $u b=U B_{1}$. 
2. Hatice Calik, Martine Labbé, and Hande Yaman. p-Center Problems, pages 79-92. Springer International Publishing, Cham, 2015.

3. Hatice Calik and Barbaros C. Tansel. Double bound method for solving the p-center location problem. Computers $\&$ Operations Research, 40(12):2991-2999, 2013.

4. Mark S. Daskin. Network and discrete location analysis. ed: John Wiley and Sons, New York, 1995.

5. Sourour Elloumi, Martine Labbé, and Yves Pochet. A new formulation and resolution method for the p-center problem. INFORMS Journal on Computing, 16(1):84-94, 2004.

6. Daniele Ferone, Paola Festa, Antonio Napoletano, and Mauricio G. C. Resende. A new local search for the p-center problem based on the critical vertex concept. In Roberto Battiti, Dmitri E. Kvasov, and Yaroslav D. Sergeyev, editors, Learning and Intelligent Optimization, pages 79-92, Cham, 2017. Springer International Publishing.

7. Daniele Ferone, Paola Festa, Antonio Napoletano, and Mauricio G. C. Resende. On the fast solution of the p-center problem. In 2017 19th International Conference on Transparent Optical Networks (ICTON), pages 1-4, July 2017.

8. David Sayah and Stefan Irnich. A new compact formulation for the discrete p-dispersion problem. European Journal of Operational Research, 256(1):62-67, 2017. 